Tropical Journal of Pharmaceutical Research December 2010; 9 (6): 605-613

(C) Pharmacotherapy Group,

Faculty of Pharmacy, University of Benin,

Benin City, 300001 Nigeria.

All rights reserved.

Review Article

Available online at http://www.tjpr.org

\title{
Malignant Pleural Mesothelioma Prognostic Marker: A Review of Osteopontin and Soluble Mesothelin Peptides
}

\author{
Franca N Alaribe, Staffania Maniero and Cecilia Pancaldi
}

Department of Morphology and Embryology, Section of Cell Biology and Molecular Genetics, University of Ferrara, Via Fossato di Mortara 64/b, 44100 Ferrara, Italy.

\begin{abstract}
This article is a review of a series of three studies that proved the involvement of osteopontin as a prognostic marker in malignant pleural mesothelioma (MPM) cancers. The approach used involved synthesizing and analysing the three articles. The first proves the utilization of osteopontin and mesothelin for diagnostic and prognostic assessment of MPM in patients previously exposed to asbestos and those with pleural metastasis. The second proves that immunohistochemical analysis identified osteopontin to have a prognostic role from its expression in MPM. The third uses the specificity and sensitivity of serum osteopontin to distinguish patients with plueral mesothelioma from subjects with asbestos-related non-malignant diseases. Overall, these remarkable findings are important for understanding of expression of osteopontin in malignant mesothelial cells and in patients with MPM. The use of osteopontin as a prognostic marker in MPM is promising and such fine molecular studies certainly help in bringing osteopontin closer to clinical practice.
\end{abstract}

Keywords: Osteopontin, Mesothelioma, Prognostic marker. 


\section{INTRODUCTION}

Asbestos is the major cause of malignant mesothelioma (MM) in the Western world $[1,2]$. In spite of the large number of individuals exposed to asbestos, only a relatively small percentage of them, during their lifetime, will develop $\mathrm{MM}$, leading some experts to suggest that there are additional factors that predispose to the disease. Simian virus 40 (SV40), a DNA virus, has been implicated as a carcinogen in the formation of mesotheliomas. Recent studies have shown that SV40 sequences could be found in up to $60 \%$ of mesotheliomas and that $100 \%$ of the infected cells express SV40 T antigen (Tag); however, the infected cells are not lysed by SV40 due to abnormally high levels of p53 expression in mesothelial cells which binds Tag and limits the replication of SV40. This probably sets up a situation where the mesothelial cells infected with SV40 can acquire genetic mutations and a malignant phenotype [3-6].

\section{Malignant pleural mesothelioma}

Malignant mesotheliomas are tumours that arise from the mesothelial cells of serosal surfaces such as the pleura and peritoneum. The single term, malignant pleural mesothelioma (MPM), can be misleading in that these tumours present with substantial phenotypic variability and are, therefore, classified according to the relative proportions of epithelial and spindle cells [7]. The median survival rate after the diagnosis of pleural mesothelioma is 9 to 12 months; in advanced cases, resection of the tumour can prolong survival by about 3 months $[8,9]$. Mesothelioma is an extremely aggressive tumour that is highly resistant to chemotherapy and radiotherapy. Thus, significant advances in the treatment of MPM will involve an early diagnosis to select candidates for therapy with curative intent [10]. The discovery of a marker that would permit an earlier diagnosis could lead to an increase in the proportion of patients diagnosed with early-stage mesothelioma in which a multimodal treatment, including surgery and radiotherapy/chemotherapy, would eventually result in a better outcome. To date, there is no recognized marker for the diagnosis of mesothelioma or for screening of at-risk asbestos-exposed individuals. Retrospective studies of small numbers of patients with pleural mesothelioma have attempted to identify biomarkers that predate symptoms in a highrisk population. These markers include tissue polypeptide antigen, carcino-embryonic antigen, hyaluronic acid and ferritin, hyaluronic acid alone, cytokeratins such as soluble cytokeratin 19 fragment, 12-16 CA125 , and soluble mesothelin-related protein $[11,12]$.

\section{Osteopontin origin}

Osteopontin (OPN) is in the family of five small integrin-binding ligand $\mathrm{N}$-linked glycoproteins (SIBLINGs1) and a glycophosphoproteins that was first identified in 1986 in osteoblasts. Osteopontin has come to be an emerging group of molecular tools that cancer cells use to facilitate their expansion. In normal tissues, OPN interacts with a variety of integrins, including av $\beta 1$, $\alpha v \beta 3, \alpha v \beta 5, \alpha 4 \beta 1, \alpha 8 \beta 1$ and $\alpha 9 \beta 1$ as well as CD44 splice variant. It also exhibits reduced serine/theonine phosphorylation by casein kinase which induces the adhesion of human breast cancer cells almost six-fold more than hyperphosphorylated OPN; this highlights the possible modifying roles of the many posttranslational events on OPN functions. It has been shown to play an important role in tumour progression and invasion in breast, lung, colon, stomach, mesothelioma and postrate cancer $[13,15]$.

Osteopontin has been seen as a promising biomarker due to its expression on geneexpression arrays to predict survival and recurrence patterns in patients with pleural mesothelioma $[16,18]$ and it seems especially interesting as a potential early diagnostic marker because it has been shown to differentiate asbestos-exposed patients from 
stage I mesothelioma patients. However, questions are always raised about the clinical utility of this marker especially when the report did not include other pleural malignancies and nonmalignant asbestosinduced pleural diseases as controls

$[19,20]$.

This review highlights a series of three recent studies/articles that prove the use of osteopontin as a diagnostic and a marker in malignant pleural mesothelioma. The first article evaluates the diagnostic and prognostic value of osteopontin and soluble mesothelin-related peptides measured both in blood and pleural fluids in a series of patients suspected to have MPM. The second proves that osteopontin plays a prognostic role in its immunohistochemical expression levels, which was significantly reduced in long-surviving patients with mesothelioma and vice versa. The third article identifies osteopontin as a useful biomarker in pleural mesothelioma by comparing serum levels of osteopontin in a group of subjects with asbestos-related nonmalignant disease with preoperative levels in patients with surgically-treated pleural mesothelioma $[10,14,17]$.

\section{Osteopontin as a prognostic and diagno- stic assessment tool for MPM}

Involvement of osteopontin as a prognostic marker in malignant pleural mesothelioma (MPM) has come under intense scrutiny in recent years. Bogdan et al [17] in their work to measure the diagnostic value of osteopontin both in blood and pleural fluid, recruited 112 healthy subjects previously exposed to asbestos ( $A E$ group) and 43 patients with pleural metastasis of adenocarcinomas (Mets group), 33 patients with benign pleural lesions associated with asbestos exposure (BPLAE group), and 96 patients with MPM. They found that there was low serum osteopontin in the AE group. Higher serum osteopontin levels were found in patients with BPLAE, MPM and Mets patients (Fig 1A). Interestingly, the authors discovered no statistically significant difference in serum osteopontin between the three groups of patients (Figs $1 \mathrm{~A}$ and $\mathrm{B}$ ) \{MPM, Mets, and BPLAE; $p>0.38$ for all comparisons\}. Serum osteopontin has a good ability to distinguish between patients with MPM and asbestos-exposed healthy subjects (AE) with an area under the curve (AUC) of 0.724 (95\% confidence interval $(\mathrm{Cl})$, range: 0.650 - 0.798), as shown in Fig 2. Serum osteopontin showed also a significant ability to distinguish patients with any pleural involvement (MPM, Mets or BPLAE) and healthy asbestos-exposed subjects (AE; AUC $0.735 ; 95 \% \mathrm{Cl}$, range: $0.677-0.793$ ) and patients with malignant pleural involvement (MPM or Mets) from patients with a benign pleural involvement.
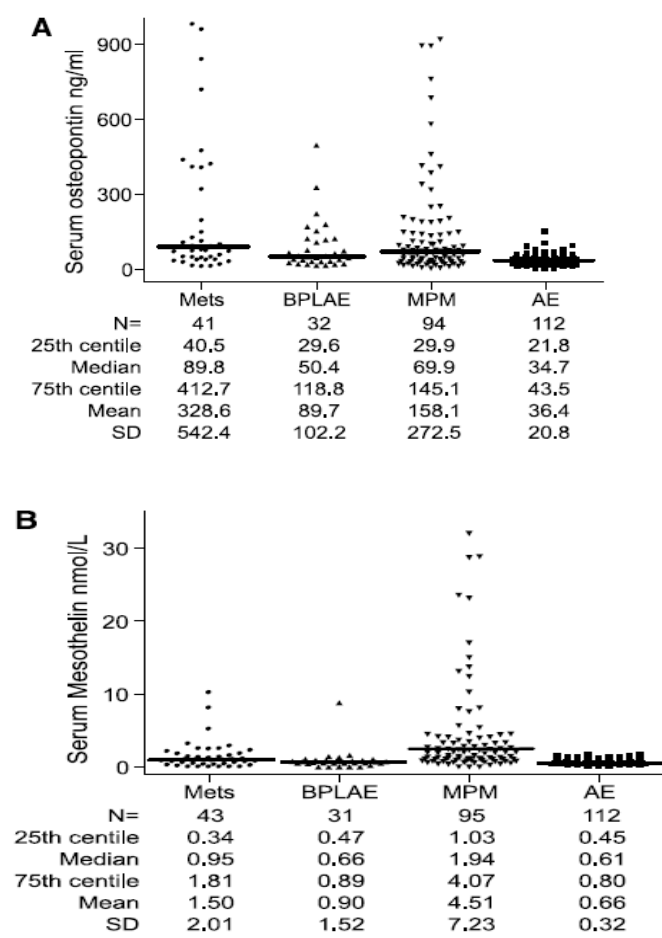

Figure 1: Diagnostic value of serum osteopontin and mesothelin in patients with MPM. (A) shows serum osteopontin values in MPM patients compared with pleural metastasis (Mets), benign pleural lesions associated with asbestos exposure (BPLAE), and healthy asbestos-exposed subjects (AE). B shows serum mesothelin in same patients. Horizontal lines, median values ( $A$ and $B)$. 


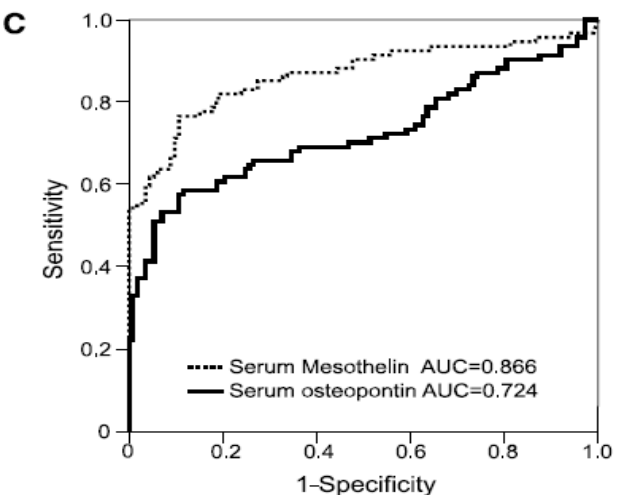

Figure 2: Diagnostic value of serum osteopontin and mesothelin in patients with MPM. C indicates ROC curve for mesothelin and osteopontin when differentiating between MPM and healthy asbestos-exposed subjects (AE group).

The diagnostic value was low in both $A E$ and BPLAE groups and slightly higher in Mets patients (see Fig 1B). MPM patients depicted higher values of mesothelin. A statistically significant correlation between serum mesothelin and blood (serum or plasma) osteopontin values were found $(p<0.02)$ in all subgroups of patients (MPM, BPLAE and Mets).

Furthermore, osteopontin and mesothelin were compared as prognostic factors. It was discovered that median survival was lower in Mets group than in MPM group and that neither age, sex nor preural value of mesothelin and osteopontin were related to survival. However, a significant relationship with survival was found for serum mesothelin as well as serum and plasma osteopontin. Patients with a high mesothelin level had a median survival of 7 months compared with 19 months for the low mesothelin level group. Similarly, patients with high serum osteopontin had a significantly shorter survival rate than patients with low serum osteopontin level.

Osteopontin serum level was related to duration of asbestos exposure and showed a good ability to discriminate between asymptomatic asbestos-exposed individuals and early stage mesothelioma patients. The most interesting result of this report is the potential usefulness of both serum osteopontin and serum mesothelin as prognostic markers in MPM.

\section{Immunohistochemical analysis and the prognostic role of osteopontin in MPM}

Sussana et al, in their investigation on the prognostic role of osteopontin expression in MPM using immunohistochemical analysis, discovered that osteopontin reactivity was specifically localized in internal control cells (i.e., macrophages)[14]. No immunoreactivity was observed in normal mesothelial cells obtained from patients with pneumothorax or in the surrounding stroma. Conversely, hyperplastic or reactive mesothelial cells in the cases of nonmalignant chronic pleuritis showed weak immunoreactivity. Occasional mesenchymal and inflammatory cells in the extracellular matrix were positive for osteopontin.

Osteopontin expression was observed in almost all MPM cases. Neoplastic cells had variable patterns of cytoplasmic reactivity, as diffuse granular or perinuclear spots, with intensity ranging from weak to intense. The percentage of distribution varied from 1 to 98 $\%$; therefore, the histologic scoring (HScore) values obtained ranged from 1 to 297 scores. In many samples, osteopontin reactivity appeared to be restricted to small, very strong cytoplasmic spots, immediately adjacent to the nucleus. This staining pattern probably corresponds to osteopontin protein in the Golgi apparatus and/or secretory vesicles.

In the LS (long-term survival) group, the distribution of HScore values of osteopontin expression varied from 5 to 250 (median, 50), whereas in the SS (short term survival) group, HScore values varied from 30 to 297 (median, 180). The difference was statistically significant $(p<0.0001$; MannWhitney $U$ ), as shown in Figure $3 \mathrm{~A}$. No statistically significant difference $(p=0.3817$; 
Mann-Whitney $U$ ) was found between the pooled LS and SS groups, in which the distribution of HScore values ranged from 5 to 297 (median, 145), and the CC group (consecutive MPM group), ranging from 1 to 285 (median, 132). The two groups were therefore statistically comparable (see Figure 3B). Furthermore, survival analysis of patients revealed that the univariate analyses comparing the LS and SS groups, osteopontin expression and performance status were significant prognostic factors $(P<$ 0.00001 and $p=0.02$, respectively), whereas no other variables correlated with survival. In multivariate analysis, however, only osteopontin retained significance as an independent predictor of survival.

From the above findings, osteopontin expression was statistically correlated to MPM in a subgroup of long-surviving patients, as opposed to short surviving patients. These data indicate that increased osteopontin expression levels may be a predictive marker of survival at the time of MPM diagnosis.
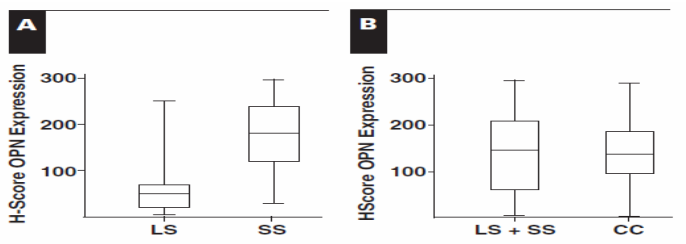

Figure 3: Distribution of osteopontin (OPN) immunohistochemical hsitologic scoring (HScore) values in malignant pleural mesothelioma (MPM). A, OPN HScore values were lower in long-term survival (LS) than in short-term survival (SS) cases $(p<.0001$; MannWhitney $\mu$ ) B, OPN Hscore values in the pooled LS and SS (LS + SS) cases were comparable to the consecutive MPM group (CC) $(p=.3817$; Mann-Whitney $\mu)$. Boxes indicate the range $\left(25^{\text {th }}-75^{\text {th }}\right.$ percentailes $)$; Whiskers, the major and minor values, and lines I the boxes, median values.

\section{Use of specificity and sensitivity of serum osteopontin as a distinguishing tool}

Harvey et al [9] undertook a study on serum osteopontin levels in patients with MPM due to exposure to asbestos, subjects with cancer but no exposure to asbestos as well as subjects without cancer and no exposure to asbestos. They discovered that the mean $( \pm S E)$ serum level of osteopontin in the entire group of subjects who were exposed to asbestos was $30 \pm 3 \mathrm{ng} / \mathrm{mL}$ (range, 2 to 221 $\mathrm{ng} / \mathrm{mL} ; 95 \% \mathrm{Cl}$, which did not differ significantly from that in subjects without exposure to asbestos $(20 \pm 4 \mathrm{ng} / \mathrm{mL}, p=$ $0.06)$. The levels in age-matched controls with no exposure to asbestos and normal radiographs did not differ significantly according to age from those in the group exposed to asbestos. Fig 4B shows that in the group with exposure to asbestos, there were no significant differences in osteopontin levels according to sex $(p=0.19)$ or the presence or absence of pleural plaques $(P=0.88)$, as Fig $4 \mathrm{C}$ illustrates. The subgroup with lung fibrosis had a significantly higher mean level of osteopontin than the subgroup without fibrosis (43 vs. $23 \mathrm{ng} / \mathrm{mL}$; $95 \% \mathrm{Cl}, 7$ to $33 \mathrm{ng} / \mathrm{mL}$, respectively, with $p=0.004$ ) (Fig $4 \mathrm{D}$ ), and the mean levels were significantly higher with 10 or more years of exposure than with fewer than 10 years of exposure (34 ng/mL vs. $16 \mathrm{ng} / \mathrm{mL} ; 95 \% \mathrm{Cl}$ for the difference, 4 to $33 ; p=0.02$ ) (Fig $4 \mathrm{C}$ ). The highest levels of serum osteopontin were found in subjects who had both plaques and fibrosis (56 $\pm 13 \mathrm{ng} / \mathrm{mL}$ ). Serum osteopontin levels were significantly lower in agematched unexposed controls than in subjects with asbestos exposure and plaques and fibrosis (mean age in both groups, $64 \pm 3$ years): $14 \pm 6$ vs. $56 \pm 13 \mathrm{ng} / \mathrm{mL}(p=0.03)$.

Among the subjects with exposure to asbestos, osteopontin levels were significantly lower in subjects with a normal chest radiograph $(21 \pm 5 \mathrm{ng} / \mathrm{mL})$, subjects with plaques (23 $\pm 3 \mathrm{ng} / \mathrm{mL}$ ), and subjects with fibrosis ( $32 \pm 7 \mathrm{ng} / \mathrm{mL}$ ) than in those who had plaques and fibrosis $(56 \pm 13 \mathrm{ng} / \mathrm{mL}, p=$ $0.004)$. A multiple regression analysis that included age, duration of exposure to asbestos, presence or absence of fibrosis, presence or absence of plaques, and International Labor Organization radiography score, was performed. Only the duration of 

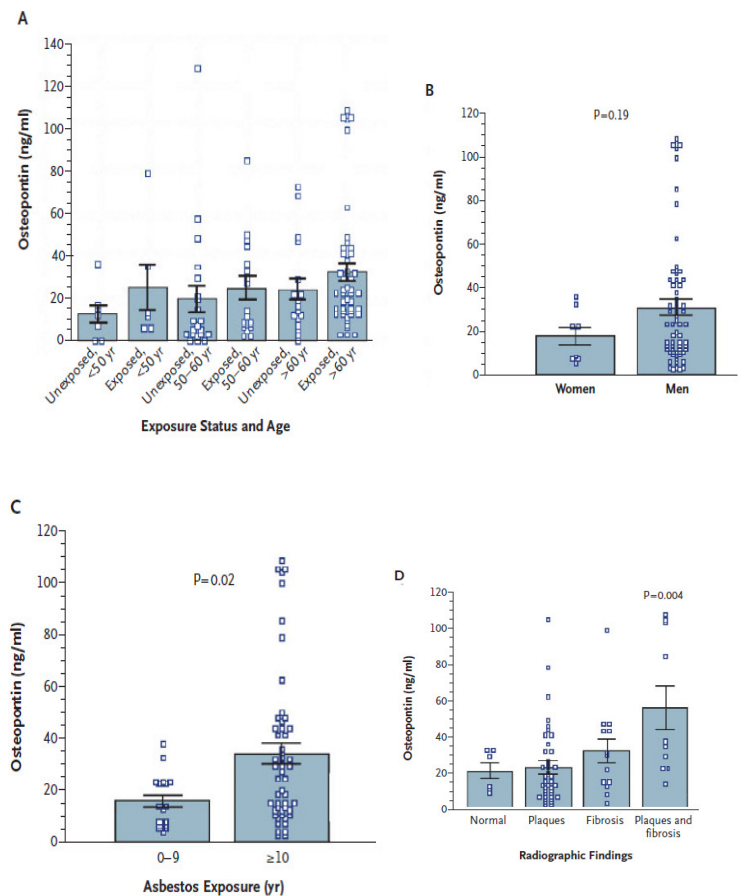

Figure 4: Mean $( \pm S E)$ serum osteopontin levels according to exposure status and age, sex, years of exposure to asbestos, and radiographic findings. There were no significant differences in osteopontin levels between subjects exposed to asbestos and age-matched controls with no exposure to asbestos for subjects according to age and year of exposure (Panel 3A). No significant differences in osteopontin levels were noted with respect to sex (Panel 3B). Osteopontin levels rose as the duration of exposure to asbestos increased (Panel $3 \mathrm{C})$ and the degree of radiographic abnormality increased (Panel 3D). The $p$ value for Panel $D$ is for the comparison of plaques and fibrosis with the other findings.

exposure to asbestos and the radiographic findings were independently associated with osteopontin levels $(p=0.001$ and $p<0.001$, respectively), with zero-order correlation coefficients of 0.357 and 0.399 , respectively.

Additionally, the mean serum osteopontin level in the group with pleural mesothelioma differed significantly from that in the group exposed to asbestos. There were no significant differences in mean serum osteopontin levels among patients with stage I mesothelioma, stage II mesothelioma or stage III mesothelioma. However, the means in all these stages differed significantly from the mean in the group exposed to asbestos $(30 \pm 3 \mathrm{ng} / \mathrm{mL}$; range, 2 to $221 \mathrm{ng} / \mathrm{mL} ; 95$ $\% \mathrm{Cl}$, with $p<0.001)$. Moreover, serum osteopontin levels in the subjects with exposure to asbestos and plaques and fibrosis differed significantly from those in the patients with pleural mesothelioma. Mean osteopontin levels were similar in men and women with mesothelioma and did not vary according to the histologic characteristics of the tumour or the history of asbestos exposure.

In their immunohistochemical findings [9], the authors showed that out of the 38 of the 76 patients with mesothelioma for whom osteopontin staining was carried out on tumour tissue, 36 (out of the 38 samples) were positive for osteopontin. They showed cytoplasmic staining in at least $50 \%$ of tumour cells, and staining intensity ranged from 1 in the case of 13 samples to 3 in the case of 15 samples; 8 samples had a staining intensity of 2. Osteopontin was seen in all pleural mesothelioma variants: 19 of 20 epithelial tumour 15 of 16 mixed tumours, and 2 of 2 sarcomatoid tumours. Lung parenchyma and adjacent normal pleura were negative for osteopontin. Fibroblasts in tumour-associated stroma were infrequently weakly positive, and the media and intima of vessels showed weak positivity.

The above study shows the apparent ability of an enzyme-linked immunosorbent assay (ELISA) for osteopontin to identify early pleural mesothelioma (fig 5E, 5F -stage I). This finding, if confirmed, would have immediate clinical applications because the use of therapy could potentially influence survival among patients with stage I pleural mesothelioma. Immunohistochemical analysis showed that osteopontin was present in the tumour cells of pleural mesothelioma but not in the stroma. The sensitivity and specificity of serum osteopontin for distinguishing patients with pleural mesothelioma from subjects with asbestors related non malignant diseases revealed an approach of 0.9 specificity of 

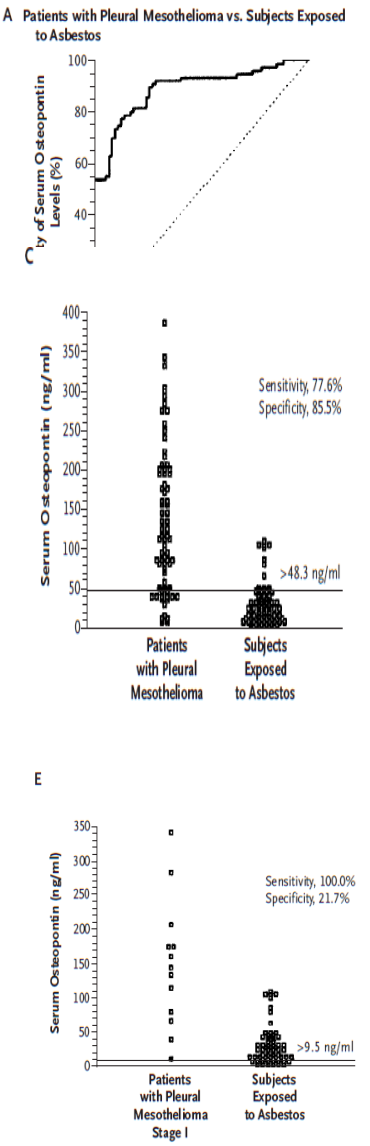

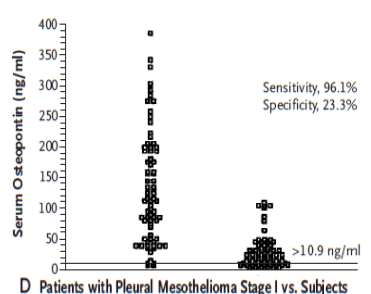

Patients with Pleural Mesothelioma Stage I vs. Subjects
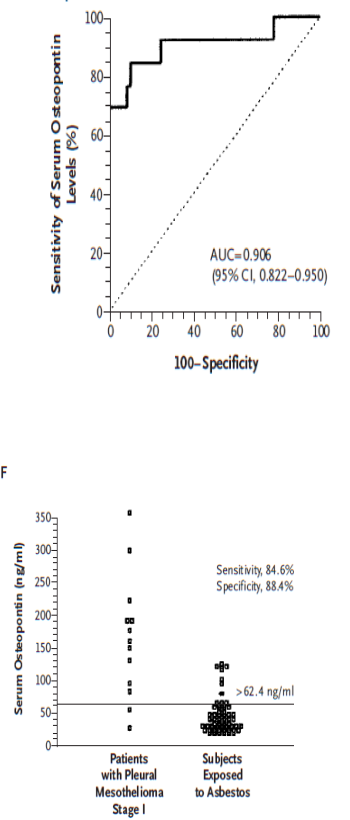

Figure 5: Sensitivity and specificity of serum osteopontin for distinguishing patients with pleural mesothelioma from subjects with asbestos-related nonmalignant disease. In Panel 4A, an analysis that included all 76 patients with pleural mesothelioma and 69 subjects with exposure to asbestos revealed an area under the curve (AUC) of $0.888(95 \% \mathrm{Cl}$, range 0.826 to 0.934$)$. In Panel $4 \mathrm{~B}$, the use of a cutoff value of $10.9 \mathrm{ng}$ of osteopontin per $\mathrm{mL}$ showed a high sensitivity for the early detection of mesothelioma. In Panel $4 \mathrm{C}$, the use of a cutoff value of $48.3 \mathrm{ng}$ of osteopontin per $\mathrm{mL}$ to minimize the total classification error had a sensitivity of $77.6 \%$. In Panel $4 \mathrm{D}$, an analysis that included the 13 patients with stage I mesothelioma and the 69 subjects with exposure to asbestos revealed an AUC of 0.906. In Panel E, the use of a cutoff value of $9.5 \mathrm{ng}$ of osteopontin per $\mathrm{mL}$ showed a high sensitivity for the early detection of stage I mesothelioma. In Panel 4F, the use of a cutoff value of $62.4 \mathrm{ng}$ of osteopontin per $\mathrm{mL}$ to minimize the total classification error had a sensitivity of $84.6 \%$ for stage I pleural mesothelioma.

area under curve (AUC). These findings provide support for the specificity of osteopontin as a marker for transformed mesothelial cells.

Furthermore, the above data suggest that serum osteopontin levels could be used to discriminate between persons with exposure to asbestos who do not have early pleural mesothelioma and those with exposure to asbestos who do have early pleural mesothelioma, regardless of the histologic type of the mesothelioma. Osteopontin levels, however, are also elevated in other types of cancers, including gastrointestinal, laryngeal, and urinary neoplasms, and these cancers have been weakly associated with exposure to asbestos.

\section{Consequences and advantages of osteopontin as a marker in MPM}

The median survival after diagnosis of pleural mesothelioma is 9 to 12 months [8-9]. In advanced cases, resection of the tumour can prolong survival by about 3 months. Patients with stage IA disease, however, can survive for five or more years if the tumour is promptly resected. Unfortunately, the difficulty in detecting early disease means that less than 5 percent of patients with pleural mesothelioma present with stage IA disease. Therefore, a marker or series of biomarkers that can predict the development of mesothelioma or detect pleural mesothelioma in its early stages in populations with exposure to asbestos would be of considerable value.

Osteopontin has been pin-pointed as an early marker for MPM diagnosis by a series of recent studies, although Bodgan et al [17] pointed out that the use of osteopontin as an MPM screening marker is expected to be difficult because the incidence of the disease is very low, even in asbestos-exposed population. Consequently, most patients with a positive test will, infact, be false positive but at least, osteopontin could be used as a first step selection marker combined with a more 
specific assay in the subgroup of osteopontin positive patients. Furthermore, the complexity of osteopontin as a molecule and its several distinct forms should also be considered. Bogdan et al [17] found mesothelin to be a better MPM diagnostic marker than osteopontin in non-epitheloid MPM but despite good specificity, the poor sensitivity of mesothelin makes it insufficient for use as a unique screening marker. Notwithstanding, the results depend on the choice of the assay used. Therefore, future quantification of other soluble isoforms of osteopontin could possibly give better results. Interestingly, Bogdan et al [17] discovered osteopontin to be a useful potential prognostic marker in MPM. Similarly, Sussana et al [14] discovered osteopontin to be a significant prognostic factor from its expression and performance status on survival and multivariate analysis of patients. This prognostic role of osteopontin demonstrated in MPM patients is independent of other prognostic factors such as sex, age, stage and histologic type, though its serum measurement level was of low diagnostic usefulness owing to the lack of specificity; nevertheless, its expression was statistically correlated with a subgroup of long-surviving patients with MPM, as opposed to patients with short-term survival. These data indicate that increased osteopontin expression levels may be a predictive marker of survival at the time of MPM diagnosis. Consequently, more aggressive initial treatment targeting the osteopontin protein with antibodies and immunotherapeutic strategies may be envisaged.

Furthermore, the most important finding by Harvey et al was the apparent ability of an enzyme-linked immunosorbent assay (ELISA) for osteopontin to identify early pleural mesothelioma (stage 1). This makes osteopontin a potential factor for immediate clinical application in the identification of patients with stage 1 pleural mesothelioma. Combination of radiographic findings and serum levels of osteopontin could be used to stratify the risk of pleural mesothelioma in populations with exposure to asbestos. Immunohistochemical analysis supported osteopontin as a marker for transformed mesothelioma cells because of its expression on the tumor cells of pleural mesothelioma but not in the stroma.

Harvey et als [9] data suggest that serum osteopontin levels could be used to discriminate between persons with exposure to asbestos who do not have early pleural mesothelioma and those with exposure to asbestos who have early pleural mesothelioma, regardless of the histologic type of the mesothelioma. Moreover, the fact that area under the curve (AUC) approached 0.9 suggests that osteopontin level has a positive predictive power equivalent to that of CA-125 for ovarian cancer (Fig 4A). In addition, osteopontin level has been elevated in other cancers, such as laryngeal, urinary neoplasm, gastrointestinal cancers, which have been weakly associated with exposure to asbestos.

\section{CONCLUSION}

One can suggest that asbestos workers with high osteopontin levels who do not appear to have mesothelioma should be evaluated to rule out the presence of other cancers. Mesothelioma histologic subtype and blood levels of mesothelin and osteopontin were independent prognostic factors for survival because they probably reflect different aspects of tumour biology. This is also sustained by the absence of tight correlations between values of osteopontin and mesothelin. Further studies are needed to explain how mesothelin and osteopontin are produced, secreted, and involved in mesothelioma tumour progression. Kinetic studies will also be important to evaluate the value of these markers in monitoring patient response to therapy.

Since mesothelioma is known to be an extremely destructive tumour and is highly resistant to chemotherapy and radiotherapy, significant advances in treatment of MPM will 
mean early diagnosis to select candidates for therapy with curative intent. More research work should be undertaken to improve on the specificity of osteopontin as a marker that would permit an earlier diagnosis could lead to an increase in the proportion of patients diagnosed with early-stage mesothelioma in which a multimodal treatment, including surgery and radiotherapy/chemotherapy, would eventually result in a better outcome.

Furthermore, this review strongly suggests that detection of osteopontin levels among a cohort of individuals exposed to asbestos could represent a useful prognostic marker to identify those at higher risk for MM with the result that this subgroup of high risk individuals could be closely monitored for early detection and possibly restorative surgical elimination.

\section{REFERENCES}

1. Hassan R, Remaley AT, Sampson ML. Detection and quantitation of serum mesothelin, a tumor marker for patients with mesothelioma and ovarian cancer. Clinical Cancer Res 2006; 12: 447-453.

2. Bianchi C, Bianchi T. Malignant mesothelioma: global incidence and relationship with asbestos. Ind Health 2007; 45: 379-387.

3. Rizzo P, Bocchetta M, Power A. SV40 and the pathogenesis of Mesothelioma. Cancer Biol 2001; 11: 63-71.

4. Shoshana J Weiner, Siyamek Neraji-Miandoab. Pathogenesis of Mlignant Pleural Mesothelioma and the Role of Environmental and Genetic Factors. J Carcinog 2008; 7: 3-7

5. Cecilia $P$, Veronica $B$, Roberto $G$, Francesca $V$, Alfredo C, Fernanda M, Luciano M, Mauro Togno. Simian Virus 40 Sequences in Blood Specimens From Healthy Individuals of Casale Monferrato, An Industrial Town With A History of Asbestos Pollution. Journal of Infection 2009; 38: 53-60.

6. Alfonso C, Rudy F, Agnese V, Rodolfo B, Vittorio G, Giovanni G, Francesca C, Franco O, Eugenio $C$, Roberta L, Mauro T et al. SV4O Enhances the Risk of Malignant Mesothelioma among People Exposed to Asbestos: A Molecular Epidemiologic Case-Control Study. Cancer Res 2005; 65(8): 3049-3052.
7. Neragi-Miandoab S. Multimodality approach in management of malignant pleural mesothelioma. Eur J Cardiothorac Surg 2006; 29(1): 14-19.

8. Vogelzang NJ, Rusthoven JJ, Symanowski J. Phase III study of pemetrexed in combinationwith cisplatin versus cisplatin alone in patients with malignant pleural mesothelioma. $J$ Clin Oncol.2003; 21: 2636-2644.

9. Harvey I, Dan L, Fulvio L, Michael H, Zhandong Liu, Naimei Tang, Michele C, Craig Webb, Anil W. Asbestos Exposure, Pleural Mesothelioma, and Serum Osteopontin Levels: Engl J Med 2005; 353: 1564-1573.

10. Robinson BW, Musk AW, Lake RA. Malignant mesothelioma. Lancet 2005; 366: 397-408.

11. O'Regan A, Berman JS. Osteopontin: a key cytokine in cell-mediated and granulomatous inflammation. Int J Exp Pathol 2000; 81: 373390.

12. Onda $M$, Nagata $S$, Ho M. Megakaryocyte potentiation factor cleaved from mesothelin precursor is a useful tumor marker in the serum of patients with mesothelioma. Clin Cancer Res 2006; 12: 4225-4231.

13. Akeila Bellahcene. Small Integrin-Binding Ligand NLinked Glycoprotein (Siblings): Multifunctional Proteins in Cancer. Nat Rev Cancers 2008; 8 (3) : 212-226.

14. Susanna $C$, Luisella $R$, Dario $M$, Paolo $C$, Elisa $B$, Francesco A, Luca M, Giorgio V, Mauro $P$. Prognostic Role of Osteopontin Expression in Malignant Pleural Mesothelioma. Am J Clin Pathol 2008; 130: 58-64.

15. Antonio Macri. Role of Osteopontin in Breast Cancer Patients. Tumori 2009; 95: 48-52

16. Pass HI, Liu Z, Wali A. Gene expression profiles predict survival and progression of pleural mesothelioma. Clin Cancer Res 2004; 10: 849859.

17. Bogdan-DG, Arnaud S, Patrick D, Bachar C, Marc L, Pierre L, Marc G, Henri P, Marie-C, Philippe L. Utility of Osteopontin and Serum Mesothelin in Malignant Pleural Mesothelioma Diagnosis and Prognosis Assessment. Clin Cancer Res 2007; 13(10): 15.

18. Shiomi $K$, Miyamoto $H$, Segawa T. Novel ELISA system for detection of $N-E R C /$ mesothelin in the sera of mesothelioma patients. Cancer Science 2006; 97: 928-932.

19. Cullen MR. Serum osteopontin levels is it time to screen asbestos-exposed workers for pleural mesothelioma? N Engl J Med 2005; 353: 1617-1628.

20. Serlin D, O'Regan AW, Berman JS. Asbestos exposure and serum osteopontin. $N$ Engl $J$ Med 2006; 354(2 Pt 1): 304-305. 